\title{
(2) OPEN ACCESS \\ Ivabradine added to usual care in patients with heart failure: a systematic review with meta-analysis and trial sequential analysis
}

\section{Mathias Maagaard (으, , ${ }^{1}$ Emil Eik Nielsen 다 , ${ }^{1,2}$ Naqash Javaid Sethi 다, ${ }^{1}$ Ning Liang 다 , ${ }^{3,4}$ Si-Hong Yang (ㅇ) ,4 Christian Gluud (1) , ${ }^{1,5}$ Janus Christian Jakobsen (1) ${ }^{1,5}$}

\subsection{6/bmjebm-2021-111724}

- Additional supplemental material is published online only. To view, please visit the journal online (http:// dx.doi.org/10.1136/ bmjebm-2021-111724).

For numbered affiliations see end of article.

\section{Correspondence to:} Dr Mathias Maagaard, Copenhagen Trial Unit, Centre for Clinical Intervention Research, Copenhagen University Hospital, Copenhagen, The Capital Region of Denmark, Denmark; mathias.maagaard@ctu.dk

Check for updates

( $)$ Author(s) (or their employer(s)) 2021. Re-use permitted under CC BY-NC. No commercial re-use. See rights and permissions. Published by BMJ.

To cite: Maagaard M, Nielsen EE, Sethi NJ, et al. BMJ Evidence-Based Medicine Epub ahead of print: [please include Day Month Year]. doi:10.1136/ bmjebm-2021-111724

\section{Abstract}

Objectives To assess the beneficial and harmful effects of adding ivabradine to usual care in participants with heart failure.

Design A systematic review with meta-analysis and trial sequential analysis.

Eligibility criteria Randomised clinical trials comparing ivabradine and usual care with usual care (with or without) placebo in participants with heart failure.

Information sources Medline, Embase, CENTRAL, LILACS, CNKI, VIP and other databases and trial registries up until 31 May 2021.

Data extraction Primary outcomes were all-cause mortality, serious adverse events and quality of life. Secondary outcomes were cardiovascular mortality, myocardial infarction and non-serious adverse events. We performed meta-analysis of all outcomes. We used trial sequential analysis to control risks of random errors, the Cochrane risk of bias tool to assess the risks of systematic errors and the Grading of Recommendations Assessment, Development and Evaluation (GRADE) to assess the certainty of the evidence.

Results We included 109 randomised clinical trials with 26567 participants. Two trials were at low risk of bias, although both trials were sponsored by the company that developed ivabradine. All other trials were at high risk of bias. Meta-analyses and trial sequential analyses showed that we could reject that ivabradine versus control reduced all-cause mortality (risk ratio $(\mathrm{RR})=0.94 ; 95 \% \mathrm{CI} 0.88$ to $1.01 ; p=0.09$; high certainty of evidence). Metaanalysis and trial sequential analysis showed that ivabradine seemed to reduce the risk of serious adverse events ( $R R=0.90 ; 95 \%$ CI 0.87 to $0.94 ; \mathrm{p}<0.00001$; number needed to treat $(\mathrm{NNT})=26.2$; low certainty of evidence). This was primarily due to a decrease in the risk of 'cardiac failure' ( $\mathrm{RR}=0.83$; 95\% CI 0.71 to 0.97 ; $\mathrm{p}=0.02$; NNT=43.9), 'hospitalisations' ( $R R=0.89$; $95 \%$ CI 0.85 to $0.94 ; \mathrm{p}<0.0001 ; \mathrm{NNT}=36.4)$ and 'ventricular tachycardia' $\quad(\mathrm{RR}=0.59 ; \quad 95 \% \mathrm{CI}$ 0.43 to $0.82 ; \mathrm{p}=0.001 ; \mathrm{NNT}=212.8$ ). However, the trials did not describe how these outcomes were defined and assessed during follow-up. Meta-analyses showed that ivabradine increased the risk of atrial fibrillation ( $R R=1.19 ; 95 \% \mathrm{CI}$ 1.04 to $1.35 ; p=0.008$; number needed to harm

\section{Summary box}

What is already known about this subject?

- Ivabradine is recommended in patients with symptoms of heart failure despite optimal background therapy for reducing heart failure hospitalisation in the 2017 American guidelines on heart failure.

- Ivabradine is recommended for reducing cardiovascular mortality and heart failure hospitalisation in the 2016 European guidelines on heart failure.

- A recent Cochrane review did not find evidence of a difference between ivabradine and placebo/ no intervention on cardiovascular mortality and serious adverse events.

$(\mathrm{NNH})=116.3)$ and bradycardia $(\mathrm{RR}=3.95 ; 95 \% \mathrm{CI}$ 1.88 to $8.29 ; \mathrm{p}=0.0003$; $\mathrm{NNH}=303)$. Ivabradine seemed to increase quality of life on the Kansas City Cardiomyopathy Questionnaire (KCCQ) (mean difference $(\mathrm{MD})=2.92 ; 95 \% \mathrm{CI} 1.34$ to $4.50 ; p=0.0003$; low certainty of evidence), but the effect size was small and possibly without relevance to patients, and on the Minnesota Living With Heart Failure Questionnaire (MLWHFQ) (MD=-5.28; 95\% CI -6.60 to -3.96 ; $\mathrm{p}<0.00001$; very low certainty of evidence), but the effects were uncertain. Meta-analysis showed no evidence of a difference between ivabradine and control when assessing cardiovascular mortality and myocardial infarction. Ivabradine seemed to increase the risk of non-serious adverse events.

Conclusion and relevance High certainty evidence shows that ivabradine does not seem to affect the risks of all-cause mortality and cardiovascular mortality. The effects on quality of life were small and possibly without relevance to patients on the KCCQ and were very uncertain for the MLWHFQ. The effects on serious adverse events, myocardial infarction and hospitalisation are uncertain. Ivabradine seems to increase the risk of atrial fibrillation, bradycardia and nonserious adverse events. 
Summary box

What are the new findings?

- In our systematic review, including 109 randomised clinical trials with 26567 participants, ivabradine did not seem to reduce all-cause mortality, cardiovascular mortality or myocardial infarction.

- Ivabradine seemed to decrease the risk of serious adverse events, mainly due to a reduction in cardiac failure and hospitalisations, but these outcomes were poorly defined and poorly assessed.

- The effect on quality of life was small and probably without relevance to patients.

- Ivabradine seemed to increase the risk of atrial fibrillation, bradycardia and non-serious adverse events.

\section{How might it impact clinical practice in the foreseeable future?}

- Based on the evidence, the guideline recommendations on the treatment of heart failure with ivabradine should be reconsidered.

PROSPERO registration number: CRD42018112082.

\section{Introduction}

Of all deaths worldwide, 30\% are attributable to cardiovascular disease. ${ }^{1}$ Heart failure is characterised by symptoms related to fluid retention such as peripheral oedema, breathlessness and dyspnoea. ${ }^{2}$ Heart failure can be caused by either functional cardiac disease (eg, a decrease in the function of the myocardium) or structural cardiac disease (eg, disease of the cardiac valves). ${ }^{34}$ Medical management of heart failure includes the use of beta-blockers, angiotensin receptor blockers, ACE inhibitors and diuretics (loop diuretics, thiazides and potassium-sparing diuretics). Ivabradine is a relatively new drug that was first introduced into heart failure guidelines in Europe in 2012 and in America in 2017. ${ }^{56}$

Ivabradine selectively inhibits the sinus node, thereby decreasing the heart rate. The decrease in heart rate, results in a decreased myocardial oxygen demand and an increased myocardial oxygen supply, thereby improving the mismatch seen in heart failure. ${ }^{7}$ Therefore, ivabradine might be an effective intervention in people with heart failure. ${ }^{7}$ A recently published Cochrane review assessed the beneficial and harmful effects of ivabradine in people with heart failure and included 19 trials with 19628 participants and did not find evidence of a difference between ivabradine and control in regard to cardiovascular mortality and serious adverse events. ${ }^{9}$ Another systematic review included 10 trials with 18036 participants, did not search all relevant databases, did not consider the risk of random error and did not assess the certainty of evidence using the Grading of Recommendations Assessment, Development and Evaluation (GRADE). ${ }^{10}$ To the best of our knowledge, no previous systematic review has assessed the beneficial and harmful effects of ivabradine compared with usual care (ie, placebo or no intervention) for people with heart failure, searching all relevant databases while considering the risks of both systematic errors and random errors. ${ }^{911-15}$
Methods

We described our methodology in detail in our protocol that was published before conducting the literature search. ${ }^{216}$ We reported this systematic review according to the recommendations of the Preferred Reporting Items for Systematic Reviews and MetaAnalyses guidelines. ${ }^{15}$ We included all trials comparing ivabradine with placebo or no intervention in patients with heart failure. Four authors (MM, EEN, S-HY and NL) independently searched and screened for trials published prior to 31 May 2021 in Medline, Embase, CENTRAL, LILACS, CNKI, VIP and other databases and trial registries, see supplement 1 in online supplemental file 1 for a detailed list of databases and trial registries. Detailed search strategies are presented in supplement 2 in online supplemental file 2. We included randomised clinical trials regardless of their design, the trial setting, the publication status, year, language or reporting of outcomes. Five authors (MM, EEN, NJS, NL and S-HY) worked in pairs and independently extracted data and assessed the risks of bias in the included trials. If data were missing or unclear, we attempted to contact the trial authors by email. We resolved disagreements through discussion or by consulting a third author (JCJ). ${ }^{2}$ We planned to include non-randomised studies identified during the literature search for the reporting of serious and nonserious adverse events. However, we did not identify such studies during the literature search, and we did not systematically search for such studies. Therefore, there is a risk that we have not identified and reported on all relevant serious and non-serious adverse events, especially those that are rare or only associated with longterm treatment.

We predefined three primary outcomes: all-cause mortality, serious adverse events and quality of life. We also predefined three secondary outcomes and eight exploratory outcomes. ${ }^{2}$ We used the trial results reported at maximal follow-up for all our outcomes.

We predefined several subgroup analyses for the assessment of the primary outcomes:

- Trials at high risk of bias compared with trials at low risk of bias

- Men compared with women

- Participants with a resting heart rate at or above 70 beats/min compared with below 70 beats/min.

- Trials administering ivabradine at or above median daily dose compared with below median daily dose

- Trials administering ivabradine at or above median duration compared with below median duration

\section{Assessment of risk of bias}

To assess the risks of systematic errors, we assessed the risk of bias for each included trial. The risk of bias was assessed individually by five reviewers working in pairs (MM, EEN, NJS, S-HY and NL). ${ }^{17}$ We assessed the risk of small study bias using funnel plots and funnel plot asymmetry tests. We planned to assess the risk of for-profit bias as part of the risk of bias assessment but post-hoc decided to only acknowledge for-profit bias throughout the review in line with the Cochrane Handbook. ${ }^{18}$

\section{Assessment of statistical and clinical significance}

We used Review Manager V.5.4 for all meta-analyses. ${ }^{19}$ We chose to analyse all primary and secondary outcome meta-analyses using fixed effect due to the BEAUTIFUL and the SHIFT trials accounting for more than $85 \%$ wt in all primary and secondary meta-analyses (excluding the quality of life assessment with the Minnesota Living With Heart Failure Questionnaire (MLWHFQ), 
see the Quality of life section). ${ }^{13} 2021$ Random-effects metaanalyses were also performed as sensitivity analyses. We used trial sequential analysis to control random errors (see below) and we adjusted the thresholds for statistical significance, as suggested by Jakobsen and colleagues, to control for the risks of random errors. ${ }^{11} 1322$ We used three primary outcomes and, therefore, adjusted the $p$ value to 0.025 as the threshold for statistical significance. When analysing our secondary and exploratory outcomes, we used a p value of 0.05 as the threshold for statistical significance, since these outcomes were meant to be hypothesis generating.

For continuous outcome data, we converted medians and IQRs to means and SDs and we converted SEs to SDs. Continuous outcomes were reported using mean differences (MDs) with 95\% CIs. Dichotomous outcomes were reported using risk ratios (RRs) with 95\% CIs. We visually inspected forest plots for the presence of heterogeneity and quantified heterogeneity using $\mathrm{I}^{2}$ statistics. Meta-analyses results are presented in forest plots (see supplement 5 to 12 in online supplemental file 1).

Meta-analyses might include too few participants to obtain enough statistical power for the reliable assessment of intervention effects. Even with statistically significant results, the credibility is poor when too few participants are included, and the intervention effects may be overestimated or underestimated. Trial sequential analysis calculates the required information size (the number of participants) needed to confirm or reject predefined anticipated intervention effects. ${ }^{13}$ Furthermore, trial sequential analysis expands the CIs when the accrued information size has not reached the required information size. Trials included in metaanalyses might introduce heterogeneity, which is also accounted for in trial sequential analysis by increasing the required information size with increasing heterogeneity. ${ }^{11}$ In an empirical review, false positive results were present in 7 out of 100 of Cochrane meta-analyses with a total of 14 false-positive metaanalytic results. Trial sequential analysis would have prevented 13 of those, had it been implemented. ${ }^{23}$ Trial sequential analysis reduces the risk of false positive results and inaccurate effect estimates in systematic reviews of interventions. ${ }^{22}$ We reported the Trial Sequential Analysis adjusted-confidence intervals (CIs) that accounts for the uncertainty of the effect when the accumulating data in the meta-analysis had not yet reached the required information size. We also reported trial sequential analysis-adjusted CIs, if the cumulative Z-curve crossed any of the trial sequential analysis boundaries of either benefit, harm or futility.

To assess the impact of missing data, we used 'best-worst case' and 'worst-best case' analyses. ${ }^{17}$ We used GRADE to assess the certainty of evidence. ${ }^{24}{ }^{25}$ We downgraded the certainty of evidence by two levels due to imprecision in GRADE if the accrued number of participants was below 50\% of the diversity-adjusted required information size (DARIS) and by one level if the accrued number of participants was between 50\% and 100\% of DARIS. We did not downgrade if the cumulative Z-curve crossed the monitoring boundaries for benefit, harm or futility, or the DARIS was reached.

\section{Results}

From our literature search, we identified 4192 records. Additionally, 11 trials were identified from other sources. After the removal of duplicates, a total of 2539 records remained. We excluded a total of 2194 records based on their title or abstract. We excluded another 236 records based on their full text, see supplement 3 in online supplemental file 1 . Therefore, we included a total of 109 clinical trials randomising 26567 participants. ${ }^{20} 21$ 26-132 Eighteen trials compared ivabradine with

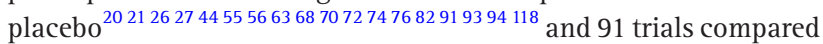
ivabradine with 'no intervention' of the 91 trials comparing ivabradine with 'no intervention', 48 trials used guideline-based therapy in both groups, ${ }^{28} 3032-3638-40485160-62646667697375777880$ 84-8789 92 95-99 10110310911211311511612012212312512813237 trials used various beta-blockers at an equal dose in both groups other than guideline-based therapy, $2931414345-474950$ 52-54 57-59 7181838890100 $102104106-108110111114117119121124126127129131 \quad 1$ trial used cyclic AMP analogue other than guideline-based therapy, ${ }^{79} 4$ trials used levosimendan other than guideline-based therapy ${ }^{42} 65105130$ and 1 trial used trimetazidine other than guideline-based therapy. ${ }^{37}$ See online supplemental file 2, baseline characteristics.

The BEAUTIFUL and the SHIFT trials contributed with more than $85 \%$ wt in all primary and secondary outcome metaanalyses. ${ }^{20}{ }^{21}$ For both trials, we identified methodological limitations. First, neither of the trials were adequately registered prior to randomising the first participants in 2004 and 2006, respectively. ${ }^{20} 21$ 133-136 Therefore, it was not adequately documented that the methodology used in the trials, including some outcomes and participating centres, was predefined. Second, primarily based on the results of these two trials, we found indications of a beneficial effect of ivabradine when assessing serious adverse events (see the Results section), primarily due to ivabradine decreasing the risk of 'cardiac failure' and 'hospitalisations' (see the Serious adverse events section). However, in the two trials, it was not described how 'cardiac failure' and 'hospitalisation' were assessed during follow-up or how 'cardiac failure' and 'hospitalisation' were defined. In the BEAUTIFUL trial, all-cause hospitalisation was not reported, which raises concerns of selective outcome reporting. ${ }^{20}$ Third, in the SHIFT substudy assessing quality of life using the Kansas City Cardiomyopathy Questionnaire (KCCQ), only 1944 participants (29.9\%) of the 6505 participants analysed in the main trial were included. ${ }^{137}$ The reason was because some countries did not participate or did not have a translated version of the KCCQ, but otherwise it was unclear how this selection of participants was conducted. ${ }^{137}$ Fourth, for serious and non-serious adverse events, there were discrepancies between the data reported in the publication of the SHIFT trial when compared with the raw data presented on ClinicalTrials.gov, see supplement 11 in online supplemental file $1 .^{21}{ }^{135}$ The BEAUTIFUL and the SHIFT trials and its authors were sponsored by the company that developed ivabradine, but the trials were otherwise judged to be at low risk of bias. All other included trials were judged to be at high risk of bias, see online supplemental file 1, risk of bias. Due to these limitations, there is a risk that we overestimate the beneficial effects and underestimate the harmful effects of ivabradine. ${ }^{21617}$

See supplement 4 online supplemental file 1 for risk of bias graph and summary.

\section{Primary outcomes}

\section{All-cause mortality}

Two trial results were judged to be at low risk of bias (but at risk of for-profit bias). ${ }^{20} 21$ In trials at low risk of bias, mortality occurred in 1075 (12.3\%) of 8720 in the ivabradine groups compared with 1099 (12.6\%) of 8702 in the control groups. Meta-analysis showed no evidence of a difference between ivabradine and control on all-cause mortality $\left(\mathrm{RR}=0.98\right.$; $95 \%$ CI 0.86 to $1.10 ; \mathrm{I}^{2}=58 \%$; figure 4 in online supplemental file 1). Meta-analysis of all trials showed a similar result $(\mathrm{RR}=0.94 ; 95 \% \mathrm{CI} 0.88$ to $1.01 ; \mathrm{p}=0.09 ; 22$ trials; high certainty of evidence; figure 6 in online supplemental file 1). Visual inspection of the forest plot and $\mathrm{I}^{2}$ statistics 
$\left(I^{2}=12 \%\right)$ indicated heterogeneity that might not be important. Trial sequential analysis showed that we had enough information to reject that ivabradine reduced the risk of all-cause mortality by $15 \%(\mathrm{RR}=0.94 ; 95 \% \mathrm{CI} 0.86$ to 1.03 ; $\mathrm{p}=0.09 ; \mathrm{I}^{2}=16 \% ; \mathrm{D}^{2}=61 \%$; figure 8 in online supplemental file 1). This outcome result was judged to be at low risk of bias (but at risk of for-profit bias). Incomplete outcome data alone seemed to have the potential to influence the results. Visual inspection of the funnel plot and Harbord's test $(p=0.51)$ did not indicate funnel plot asymmetry. See summary of findings table (figure 1) and supplement 5 in online supplemental file 1 .

\section{Serious adverse events}

Serious adverse events occurred in 3393 of 10101 participants in the ivabradine groups compared with 3758 of 10043 in the control groups. Meta-analysis showed evidence of a beneficial effect of

\begin{tabular}{|c|c|c|c|c|c|}
\hline \multicolumn{6}{|l|}{$\begin{array}{l}\text { Patient or population: patients with heart failure } \\
\text { Setting: any setting } \\
\text { Intervention: Ivabradine } \\
\text { Comparison: placebo/no intervention/usual care }\end{array}$} \\
\hline \multirow[b]{2}{*}{ Outcomes } & \multirow[b]{2}{*}{$\begin{array}{l}\text { № of } \\
\text { participants } \\
\text { (studies) } \\
\text { Follow-up }\end{array}$} & \multirow[b]{2}{*}{$\begin{array}{l}\text { Certainty of } \\
\text { the } \\
\text { evidence } \\
\text { (GRADE) }\end{array}$} & \multirow[b]{2}{*}{$\begin{array}{l}\text { Relative } \\
\text { effect } \\
(95 \% \mathrm{CI})\end{array}$} & \multicolumn{2}{|c|}{ Anticipated absolute effects } \\
\hline & & & & $\begin{array}{c}\text { Risk with } \\
\text { placebo/no } \\
\text { intervention/usual } \\
\text { care }\end{array}$ & $\begin{array}{c}\text { Risk } \\
\text { difference } \\
\text { with } \\
\text { Ivabradine }\end{array}$ \\
\hline All-cause mortality & $\begin{array}{c}19257 \\
(22 \text { RCTs ) }\end{array}$ & $\begin{array}{l}\bigoplus \bigoplus \bigoplus \bigoplus \\
\text { High }^{a, b, c}\end{array}$ & $\begin{array}{c}\mathbf{R R} \mathbf{0 . 9 4} \\
(0.88 \text { to } 1.01)\end{array}$ & 134 per 1.000 & $\begin{array}{l}\mathbf{8} \text { fewer per } \\
\mathbf{1 . 0 0 0} \\
\text { (16 fewer to } 1 \\
\text { more) }\end{array}$ \\
\hline Serious adverse events & $\begin{array}{c}20144 \\
\text { (31 RCTs) }\end{array}$ & $\begin{array}{l}\oplus \oplus \bigcirc \bigcirc \\
\text { Low }_{b, c, d}\end{array}$ & $\begin{array}{c}\mathbf{R R} \mathbf{0 . 9 0} \\
(0.87 \text { to } 0.94)\end{array}$ & 374 per 1.000 & $\begin{array}{l}37 \text { fewer per } \\
1.000 \\
\text { (49 fewer to } 22 \\
\text { fewer) }\end{array}$ \\
\hline Quality of life (KCCQ) & $\begin{array}{c}1781 \\
(2 \text { RCTs) }\end{array}$ & $\begin{array}{l}\oplus \oplus \bigcirc \bigcirc \\
\text { Low b,e,f }\end{array}$ & - & & $\begin{array}{l}\text { MD } 2.92 \text { higher } \\
\text { (1.34 higher to } \\
4.5 \text { higher) }\end{array}$ \\
\hline Quality of life (MLWHFQ) & $\begin{array}{c}221 \\
\text { (4 RCTs) }\end{array}$ & $\underset{\text { very low } b, g, h}{\oplus \bigcirc 00}$ & - & & $\begin{array}{l}\text { MD } \mathbf{5 . 2 8} \text { lower } \\
(6.6 \text { lower to } \\
3.96 \text { lower })\end{array}$ \\
\hline Cardiovascular mortality & $\begin{array}{c}18738 \\
\text { (15 RCTs) }\end{array}$ & $\begin{array}{l}\bigoplus \bigoplus \bigoplus \bigoplus \\
\text { High a,b,c }\end{array}$ & $\begin{array}{c}\text { RR } \mathbf{0 . 9 8} \\
(0.90 \text { to } 1.06)\end{array}$ & 103 per 1.000 & $\begin{array}{l}\mathbf{2} \text { fewer per } \\
1.000 \\
\text { (10 fewer to } 6 \\
\text { more) }\end{array}$ \\
\hline Myocardial infarction & $\begin{array}{c}18190 \\
\text { (9 RCTs) }\end{array}$ & $\begin{array}{l}\oplus \oplus \bigcirc \bigcirc \\
\text { Low } a, c, i\end{array}$ & $\begin{array}{c}\text { RR } 1.00 \\
(0.80 \text { to } 1.24)\end{array}$ & 17 per 1.000 & $\begin{array}{l}0 \text { fewer per } \\
1.000 \\
\text { (3 fewer to } 4 \\
\text { more) }\end{array}$ \\
\hline Non-serious adverse events & $\begin{array}{c}21598 \\
\text { (49 RCTs) }\end{array}$ & $\begin{array}{l}\oplus \bigoplus \bigoplus \bigoplus \\
\text { High } a, b, c\end{array}$ & $\begin{array}{c}\text { RR } 1.10 \\
(1.07 \text { to } 1.12)\end{array}$ & 471 per 1.000 & $\begin{array}{c}47 \text { more per } \\
1.000 \\
\text { (33 more to } 57 \\
\text { more) }\end{array}$ \\
\hline
\end{tabular}

*The risk in the intervention group (and its $95 \%$ confidence interval) is based on the assumed risk in the comparison group and the relative effect of the intervention (and its $95 \% \mathrm{CI}$ ).

CI confidence interval; MD: mean difference; RR: risk ratio

GRADE Working Group grades of evidence

High certainty: we are very confident that the true effect lies close to that of the estimate of the effect

Moderate certainty: we are moderately confident in the effect estimate: The true effect is likely to be close to the estimate of the effect, but there

is a possibility that it is substantially different

Low certainty: our confidence in the effect estimate is limited: The true effect may be substantially different from the estimate of the effect

Very low certainty: we have very little confidence in the effect estimate: The true effect is likely to be substantially different from the estimate of effect

\section{Explanations}

a. The two largest trials weighing more than $85 \%$ in all primary and secondary outcome meta-analyses and its authors were sponsored by the company that developed ivabradine. Therefore, there was a substantial risk of for-profit bias. However, the two largest trials were otherwise at low risk of bias and the certainty of the evidence has not been downgraded due to risk of bias.

b. The accrued information size reached the required information size in Trial Sequential Analysis. Therefore, imprecision was not present.

c. I statistics showed no heterogeneity or heterogeneity that might not be important. Therefore, there was no inconsistency.

d. The reporting of serious adverse events was heterogeneous. The effect was mainly attributable to a reduction in hos pitalisations. However, how hos pitalisations were defined and assessed was not adequately reported or pre-defined. Therefore, the certainty of the evidence was downgraded by two.

e. The study accounting for $95 \%$ of weight in the meta-analysis excluded $70 \%$ of the participants originally included in the main study from the quality of life substudy due to "countries not participating or not having a translated version of the quality of life measure, otherwise it was unclear how this selection of participants was conducted. Therefore, we downgraded the certainty of the evidence by one due to risk of bias.

f. The individual effect estimates had high variability and $\mathrm{D}$ statistics showed substantial heterogeneity. Therefore, the certainty of evidence was downgraded by one due to inconsistency.

g. All trials were small and at high risk of bias. Therefore, the certainty of the evidence was downgraded by two due to risk of bias.

h. D statistics indicated moderate heterogeneity and two trials included an effect that was below the minimal important difference. Therefore, the certainty of the evidence was downgraded by one due to inconsistency.

i. The accrued information size was below $50 \%$ of the required information size. Therefore, severe imprecision was present and the certainty of the evidence downgraded by two.

Figure 1 Summary of findings. RR, risk ratio. RCTs, randomised clinical trials. GRADE, Grading of Recommendations Assessment, Development and Evaluation. 
ivabradine versus control on serious adverse events $(\mathrm{RR}=0.90$; $95 \%$ CI 0.87 to $0.94 ; \mathrm{p}<0.00001 ; 31$ trials; number needed to treat $(N N T)=26.3$; low certainty of evidence; figure 17 in online supplemental file 1). Visual inspection of the forest plot and $\mathrm{I}^{2}=37 \%$ indicated heterogeneity that might not be important. Trial sequential analysis showed that we had enough information to confirm that ivabradine decreased the risk of serious adverse events by $10 \%\left(\mathrm{RR}=0.90 ; 95 \% \mathrm{CI} 0.87\right.$ to $0.94 ; \mathrm{p}<0.0001 ; \mathrm{I}^{2}=37 \%$; $\mathrm{D}^{2}=85 \%$; Trial sequential analysis graph not produced due to the first trial exceeding the required information size). This outcome result was judged to be at high risk of bias. Incomplete outcome data alone did not seem to have the potential to influence the results. Visual inspection of the funnel plot and Harbord's test $(p=0.32)$ did not indicate funnel plot asymmetry. See Summary of findings table (figure 1) and supplement 6 in online supplemental file 1.

\section{Individual serious adverse events}

The 31 trials reported on 1033 individual serious adverse events. The majority of these serious adverse events were primarily reported in the BEAUTIFUL and the SHIFT trials. For all types of individual serious adverse events, we calculated RRs, 95\% CIs and $\mathrm{p}$ values.

Ivabradine may decrease the risk of the following adverse events classified as serious by the trialists: cardiac failure $\left(\mathrm{RR}=0.83 ; 95 \% \mathrm{CI} 0.76\right.$ to $0.90 ; \mathrm{p}<0.00001 ; \mathrm{I}^{2}=41 \%$; NNT=43.9; 5 trials), ventricular tachycardia $(\mathrm{RR}=0.59 ; 95 \% \mathrm{CI} 0.43$ to 0.81 ; $\mathrm{I}^{2}=0 \%$; NNT $=212.8 ; 3$ trials) and hospitalisation (RR 0.89; 95\% CI 0.85 to $0.94 ; \mathrm{p}<0.0001 ; \mathrm{I}^{2}=56 \%$; NNT=37; 17 trials).

Ivabradine may increase the risk of bradycardia $(R R=3.95$; $95 \%$ CI 1.88 to $8.29 ; \mathrm{p}=0.0003 ; \mathrm{I}^{2}=0 \%$; number needed to harm $(\mathrm{NNH})=303 ; 3$ trials).

We regarded atrial fibrillation as a serious adverse event regardless of how it was reported in the included trials. Therefore, we conducted a meta-analysis, including the highest proportion of participants with atrial fibrillation as reported in the trials. Ivabradine may increase the risk of atrial fibrillation $(\mathrm{RR}=1.17$; $95 \% \mathrm{CI}$ 1.03 to $1.32 ; \mathrm{p}=0.02 ; \mathrm{I}^{2}=0 \% ; \mathrm{NNH}=129.9 ; 10$ trials).

\section{Quality of life}

Quality of life was reported using the KCCQ in two trials, including the SHIFT trial, analysing 1781 participants. Metaanalysis showed evidence of a beneficial effect of ivabradine versus control on quality of life using the KCCQ $(\mathrm{MD}=2.92$; 95\% CI 1.34 to 4.50; $\mathrm{p}=0.0003$; low certainty of evidence; figure 27 in online supplemental figure 1). Visual inspection of the forest plot and $\mathrm{I}^{2}=86 \%$ indicated substantial heterogeneity. Trial sequential analysis showed that we had enough information to confirm that ivabradine increased the quality of life by 2.92 points (TSA graph not produced due to the first trial exceeding the required information size). This outcome result was judged to be at high risk of bias. Incomplete outcome data seemed to have the potential to influence the results. We predefined that we would consider the observed SD divided by ' 2 ' as the minimal important difference. ${ }^{2}$ In the trials using the KCCQ, the observed difference between ivabradine and control was 2.92 points at follow-up. The SD was approximately 16.8 points; hence, the minimal important clinical difference was 8.4 points. Therefore, the observed difference of 2.92 points at follow-up was only one-third of the minimal important difference.

Quality of life was reported using the MLWHFQ in 4 trials randomising 221 participants. In three trials, it was unclear whether SDs or SEs were reported and these were excluded from the analyses. ${ }^{33} 8092$ Meta-analysis showed evidence of a difference between ivabradine and control on quality of life using the MLWHFQ (MD=-5.28; 95\% CI -6.60 to -3.96 ; $<<0.00001$; very low certainty of evidence; figure 32 in online supplemental figure 1). Visual inspection of the forest plot and $\mathrm{I}^{2}=35 \%$ indicated moderate heterogeneity. Trial sequential analysis showed that we had enough information to confirm MD of 5.28 points by ivabradine ( $\mathrm{MD}=-5.28 ; 95 \% \mathrm{CI}-7.32$ to $-3.24 ; \mathrm{p}<0.0001 ; \mathrm{I}^{2}=35 \%$; $\mathrm{D}^{2}=52 \%$; figure 34 in online supplemental figure 1 ). This outcome result was judged to be at high risk of bias. Incomplete outcome data alone did not seem to have the potential to influence the results. In the trials using MLWHFQ, the observed difference between ivabradine and control was 5.28 points at follow-up. The SD was 3.70; hence, the minimal important difference was 1.85 points. The observed difference of 5.28 points was above the minimal important difference. However, the evidence was very uncertain. See Summary of findings table (figure 1) and supplement 7 in online supplemental file 1.

\section{Secondary outcomes}

\section{Cardiovascular mortality}

Two trial results were judged to be at low risk of bias (but at risk of for-profit bias). ${ }^{20} 21$ In trials at low risk of bias, cardiovascular mortality occurred in 918 (10.6\%) of 8720 in the ivabradine groups compared with 926 (10.6\%) of 8702 in the control groups. Meta-analysis showed no evidence of a difference between ivabradine and control on cardiovascular mortality (RR=0.99; 95\% CI 0.86 to $1.15 ; \mathrm{p}=0.91 ; \mathrm{I}^{2}=66 \%$; figure 39 in online supplemental file 1). Meta-analysis of all trials showed showed a similar result $(\mathrm{RR}=0.98 ; 95 \% \mathrm{CI} 0.90$ to $1.06 ; \mathrm{p}=0.58 ; 15$ trials; high certainty of evidence; figure 41 in online supplemental file 1). Visual inspection of the forest plot and $\mathrm{I}^{2}=7 \%$ indicated heterogeneity that might not be important. Trial sequential analysis showed that we had enough information to reject that ivabradine reduced the risk of cardiovascular mortality by $15 \%$ when compared with control (RR=0.98; 95\% CI 0.88 to $1.08 ; p=0.58 ; I^{2}=7 \% ; D^{2}=49 \%$; figure 43 in online supplemental file 1). This outcome result was judged to be at low risk of bias (but at risk of for-profit bias). Incomplete outcome data alone did not seem to have the potential to influence the results. Visual inspection of the funnel plot and Harbord's test $(p=0.36)$ did not indicate funnel plot asymmetry. See Summary of findings table (figure 1) and supplement 8 in online supplemental file 1 .

\section{Myocardial infarction}

Two trial results were judged to be at low risk of bias (but at risk of for-profit bias). ${ }^{20}{ }^{21}$ In trials at low risk of bias, myocardial infarction occurred in $144(1.7 \%)$ of 8709 in the ivabradine groups compared with 142 (1.6\%) of 8690 in the control groups. Metaanalysis showed no evidence of a difference between ivabradine and control on myocardial infarction $(\mathrm{RR}=1.01$; $95 \% \mathrm{CI} 0.80$ to $1.27 ; p=0.92 ; I^{2}=0 \%$; figure 49 in online supplemental file 1 ). Meta-analysis of all trials showed a similar result $(\mathrm{RR}=1.00$; 95\% CI 0.80 to $1.24 ; p=0.96$; 9 trials; low certainty of evidence; figure 50 in online supplemental file 1). Visual inspection of the forest plot and $\mathrm{I}^{2}=0 \%$ indicated no heterogeneity. Trial sequential analysis showed that we did not have enough information to reject that ivabradine reduced the risk of myocardial infarction by $15 \%$ when compared with control $(\mathrm{RR}=1.01 ; 95 \% \mathrm{CI} 0.41$ to 2.43 ; $p=0.83 ; I^{2}=0 \% ; D^{2}=0 \%$; figure 52 in online supplemental file 1 ). This outcome result was judged to be at low risk of bias (but at risk of for-profit bias). Incomplete outcome data alone seemed to have 
the potential to influence the results. See Summary of findings table (figure 1) and supplement 9 in online supplemental file 1.

\section{Non-serious adverse events}

Two trial results were judged to be at low risk of bias (but at risk of for-profit bias)..$^{21}$ In trials at low risk of bias, non-serious adverse events occurred in 5264 (60.4\%) of 8709 participants in the ivabradine groups compared with 4798 (55.2\%) of 8690 participants in the control groups. Meta-analysis showed evidence of a harmful effect of ivabradine versus control on non-serious adverse events ( $R R=1.10 ; 95 \%$ CI 1.00 to $1.21 ; p=0.05 ; I^{2}=93 \%$; figure 57 in online supplemental file 1). Meta-analysis of all trials showed a similar result $(R R=1.10 ; 95 \%$ CI 1.07 to $1.12 ; p<0.00001$; $\mathrm{NNH}=22.5 ; 49$ trials; high certainty of evidence; figure 59 in online supplemental file). Visual inspection of the forest plot and $\mathrm{I}^{2}=12 \%$ indicated heterogeneity that might not be important. Trial sequential analysis showed that we had enough information to confirm that ivabradine increased the risk of non-serious adverse events by $10 \%$ when compared with control $(\mathrm{RR}=1.10$; 95\% CI 1.07 to $1.12 ; \mathrm{p}<0.0001 ; \mathrm{I}^{2}=12 \% ; \mathrm{D}^{2}=83 \%$; figure 61 in online supplemental file 1). This outcome result was judged to be at low risk of bias (but at risk of for-profit bias). Incomplete outcome data alone did not seem to have the potential to influence the results. Visual inspection of the funnel plot and Harbord's test $(p=0.21)$ did not indicate funnel plot asymmetry. See Summary of findings table (figure 1) and supplement 10 in online supplemental file 1.

\section{Individual non-serious adverse events}

Ivabradine may increase the risk of 'bradycardia' ( $R R=1.62$; 95\% CI 1.01 to $2.60 ; \mathrm{p}=0.05 ; \mathrm{I}^{2}=45 \%$; $\mathrm{NNH}=39.4 ; 25$ trials), 'heart rate decreased' (RR=4.32; 95\% CI 3.39 to $5.50 ; \mathrm{I}^{2}=0 \%$; $\mathrm{NNH}=33 ; 3$ trials), and phosphenes ( $R R=4.71 ; 95 \%$ CI 3.67 to $6.04 ; \mathrm{p}<0.00001$; $\mathrm{I}^{2}=0 \% ; \mathrm{NNH}=33.8 ; 20$ trials).

Ivabradine may decrease the risk of 'sinus tachycardia' ( $R R=0.39 ; 95 \% \mathrm{CI} 0.27$ to $0.56 ; \mathrm{p}<0.00001 ; \mathrm{NNT}=52.4 ; 2$ trials) and 'hypotension' (RR=0.70; 95\% CI 0.55 to $0.90 ; \mathrm{I}^{2}=0 \%$; NNT=192.3; 5 trials).

\section{Exploratory outcomes}

The results of our exploratory outcomes are reported in supplement 12 in online supplemental file 1.

\section{Subgroup analyses}

We predefined several subgroup analyses for the primary outcomes. $^{2}$

When assessing all-cause mortality, test for subgroup differences ( $\mathrm{p}=0.06)$ suggested a difference between trials administering ivabradine at or above median duration ( $R R=0.95 ; 95 \% \mathrm{CI} 0.88$ to 1.02) compared with trials administering ivabradine below median duration ( $\mathrm{RR}=0.47$; 95\% CI 0.23 to 0.99 ).

When assessing serious adverse events, test for subgroup differences $(\mathrm{p}=0.005)$ suggested a difference between trials administering ivabradine at or above median duration $(\mathrm{RR}=0.92$; $95 \% \mathrm{CI}$ 0.88 to 0.95$)$ compared with trials administering ivabradine below median duration ( $\mathrm{RR}=0.53$; 95\% CI 0.36 to 0.77 ).

When assessing quality of life on the KCCQ, test for subgroup differences $(p=0.007)$ suggested a potential difference between trials administering ivabradine at or above median duration $(\mathrm{MD}=2.40$; $95 \% \mathrm{CI} 0.77$ to 4.03$)$ compared with trials administering ivabradine below median duration ( $\mathrm{MD}=12.00$; 95\% CI 5.23 to 18.77). When assessing quality of life on the MLWHFQ, test for subgroup differences $(p=0.05)$ suggested a potential difference between trials administering ivabradine at or above median duration $(\mathrm{MD}=-13.80 ; 95 \% \mathrm{CI}-23.17$ to -4.44$)$ compared with trials administering ivabradine below median duration $(\mathrm{MD}=-1.14$; 95\% CI -9.90 to 7.61 ).

See the respective supplementary sections for all-cause mortality, serious adverse events and quality of life for all subgroup analyses.

For all other subgroup analyses, test for subgroup differences did not show evidence of a difference between the subgroups or the subgroup analyses could not be conducted.

\section{Discussion}

The objective of our systematic review was to assess both the beneficial and harmful effects of adding ivabradine to usual care versus usual care with or without placebo in people with heart failure. We included 109 randomised clinical trials randomising 26567 people with heart failure. All trials were judged to be at high risk of bias, except for the BEAUTIFUL and the SHIFT trials that were judged to be at low risk of bias (but at risk of for-profit bias)..$^{12021}$ The BEAUTIFUL and the SHIFT trials accounted for more than 85\% of weight in most meta-analysis and we did, therefore, now downgrade the certainty of the evidence due to risk of bias for most outcomes. However, we downgraded the certainty of the evidence for serious adverse events due to methodological limitations regarding the reporting of serious adverse events (see second paragraph of the Results section). Our results must be interpreted in the light of the high risks of bias and risks of for-profit bias that might result in overestimation of beneficial effects and underestimation of harmful effect of ivabradine. Due to the BEAUTIFUL and the SHIFT trials contributing with more than $85 \%$ of weight in all primary and secondary outcome meta-analyses, the results and conclusions presented in this systematic review can mostly be applied to people matching the populations in the BEAUTIFUL and the SHIFT trials.

Our results showed that ivabradine does not seem to affect the risks of all-cause mortality, cardiovascular mortality and myocardial infarction. Ivabradine seemed to decrease the risk of serious adverse events, primarily due to a decrease in the risk of 'cardiac failure', 'hospitalisations' and 'ventricular tachycardia'. However, in the BEAUTIFUL and the SHIFT trials, and in the other trials reporting these outcomes, it was not described how these outcomes were assessed during follow-up or how the outcomes were defined. The effects on quality of life using the KCCQ were small and possibly without relevance to patients. The effects on quality of life using the MLWHFQ were very uncertain. Ivabradine seemed to increase the risk of atrial fibrillation, bradycardia, and non-serious adverse events. See Summary of findings table (figure 1).

Our systematic review has strengths. First, we predefined our methodology in detail in a protocol that was published prior to conducting the literature search. ${ }^{2}{ }^{16}$ Second, we identified a total of 109 trials, which is more than any other previous systematic review on the topic. This has increased our precision and, therefore, strengthened our results. The recently published Cochrane review only identified 19 trials with 19628 participants (90 trials less than ours). ${ }^{9}$ Third, we used trial sequential analysis on both primary and secondary outcomes ${ }^{11}$ and we adjusted our thresholds for statistical significance for the primary outcomes ${ }^{13}$ to control the risks of random errors. Fourth, we judged the risk of bias of all included trials to assess the risks of systematic errors. ${ }^{2425}$ 
Fifth, we used our eight-step procedure to assess if the thresholds for statistical and clinical significance were crossed. ${ }^{13}$ Moreover, we included all randomised clinical trials identified through our literature search without imposing restrictions on their publication type, status, language and their reporting of outcomes. We attempted to contact the authors of the trials if data were incomplete or additional information was needed.

Our review also has limitations. First, all the included trials were judged to be at a high risk of bias as well as having a high risk of selective outcome reporting bias and for-profit bias. ${ }^{18}$ Nine of the trials were in some way sponsored by the company that developed ivabradine, including the BEAUTIFUL and the SHIFT trials that randomised 17475 participants, accounting for more than $85 \%$ in all primary and secondary meta-analysis. ${ }^{20} 21556370748193$ Research has shown that drug trials funded by manufacturing companies tend to show more favourable efficacy results than trials funded by other sources. ${ }^{18}$ Moreover, 18 trials were reported only as abstracts which made the interpretation of methodology and results problematic. ${ }^{2628-32} 3439447391959699100138-140$ Therefore, there is a risk that our results are also biased and, therefore, overestimate the beneficial effects of ivabradine and underestimate the harmful effects. ${ }^{18141-146}$

\section{Conclusion and relevance}

High certainty evidence shows that ivabradine does not seem to affect the risks of all-cause mortality and cardiovascular mortality. The effects on quality of life were small and possibly without relevance to patients on the KCCQ and were very uncertain for the MLWHFQ. The effects on serious adverse events, myocardial infarction and hospitalisation are uncertain. Ivabradine seems to increase the risk of atrial fibrillation, bradycardia and non-serious adverse events,

\section{Differences between the protocol and the systematic review}

We conducted our literature search in parallel with another systematic review on the effects of adding ivabradine to usual care in participants with angina pectoris due to coronary artery disease ${ }^{147}$ We originally planned to analyse and report the results, including participants with coronary artery disease and participants with heart failure into one review, but due to clinical and statistical heterogeneity, we decided to report the results in two separate reviews. ${ }^{2}$

\author{
Author affiliations \\ ${ }^{1}$ Copenhagen Trial Unit, Centre for Clinical Intervention Research, \\ Copenhagen University Hospital, Copenhagen, Denmark \\ ${ }^{2}$ Department of Internal Medicine, Holbaek Sygehus, Holbaek, Denmark \\ ${ }^{3}$ Institute of Basic Research in Clinical Medicine, China Academy of \\ Chinese Medical Sciences, Beijing, China \\ ${ }^{4}$ China Academy of Chinese Medical Sciences, Beijing, China \\ ${ }^{5}$ Department of Regional Health Research, The Faculty of Health Sciences, \\ University of Southern Denmark, Odense, Denmark
}

Acknowledgements We would like to thank Liliya Ziganshina for assisting us with screening and data extracting articles in Russian.

Contributors Guarantors: MM and JCJ.MM: conceived the systematic review, conducted literature search, data extraction, data analysis and data interpretation and wrote the article. EEN, NL and S-HY: conducted literature search and data extraction and amended the article. NJS: conducted data extraction and amended the article. CG: helped conceive the systematic review, provided invaluable comments and amended the article. JCJ: conceived the systematic review, aided in data interpretation and amended the article.

Funding This study was funded by the Copenhagen Trial Unit, Centre for Clinical Intervention Research, through wages paid to JCJ and CG. The funding is through the Danish Finance Act and the funding source had no influence on the systematic review.

Competing interests None declared.

Patient consent for publication Not applicable.

Provenance and peer review Not commissioned; externally peer reviewed.

Data availability statement Data are available upon reasonable request. All data relevant to the study are included in the article or uploaded as supplementary information.

Supplemental material This content has been supplied by the author(s). It has not been vetted by BMJ Publishing Group Limited (BMJ) and may not have been peer-reviewed. Any opinions or recommendations discussed are solely those of the author(s) and are not endorsed by BMJ. BMJ disclaims all liability and responsibility arising from any reliance placed on the content. Where the content includes any translated material, BMJ does not warrant the accuracy and reliability of the translations (including but not limited to local regulations, clinical guidelines, terminology, drug names and drug dosages), and is not responsible for any error and/or omissions arising from translation and adaptation or otherwise.

Open access This is an open access article distributed in accordance with the Creative Commons Attribution Non Commercial (CC BY-NC 4.0) license, which permits others to distribute, remix, adapt, build upon this work non-commercially, and license their derivative works on different terms, provided the original work is properly cited, appropriate credit is given, any changes made indicated, and the use is non-commercial. See: http://creativecommons.org/licenses/by-nc/4.0/.

\section{ORCID iDs}

Mathias Maagaard http://orcid.org/0000-0002-9037-7295

Emil Eik Nielsen http://orcid.org/0000-0003-1108-9533

Naqash Javaid Sethi http://orcid.org/0000-0002-8025-1939

Ning Liang http://orcid.org/0000-0003-0260-2331

Si-Hong Yang http://orcid.org/0000-0002-6767-9826

Christian Gluud http://orcid.org/0000-0002-8861-0799

Janus Christian Jakobsen http://orcid.org/0000-0002-3642-2120

\section{References}

1 GBD 2013 Mortality and Causes of Death Collaborators. Global, regional, and national age-sex specific all-cause and cause-specific mortality for 240 causes of death, 1990-2013: a systematic analysis for the global burden of disease study 2013. Lancet 2015;385:117-71.

2 Maagaard M, Nielsen EE, Gluud C, et al. Ivabradine for coronary artery disease and/or heart failure-a protocol for a systematic review of randomised clinical trials with meta-analysis and trial sequential analysis. Syst Rev 2019;8:39.

3 Writing Committee Members, Yancy CW, Jessup M, et al. 2013 ACCF/ AHA guideline for the management of heart failure: a report of the American College of cardiology Foundation/American heart association Task force on practice guidelines. Circulation 2013;128:e240-327.

4 Ponikowski P, Voors AA, Anker SD, et al. 2016 ESC Guidelines for the diagnosis and treatment of acute and chronic heart failure: The Task Force for the diagnosis and treatment of acute and chronic heart failure of the European Society of Cardiology (ESC)Developed with the special 
contribution of the Heart Failure Association (HFA) of the ESC. Eur Heart J 2016;37:2129-200.

5 Ponikowski P, Voors AA, Anker SD, et al. 2016 ESC Guidelines for the diagnosis and treatment of acute and chronic heart failure: The Task Force for the diagnosis and treatment of acute and chronic heart failure of the European Society of Cardiology (ESC)Developed with the special contribution of the Heart Failure Association (HFA) of the ESC. Eur Heart J 2016;37:2129-220.

6 Yancy CW, Jessup M, Bozkurt B, et al. 2017 ACC/AHA/HFSA Focused Update of the 2013 ACCF/AHA Guideline for the Management of Heart Failure: A Report of the American College of Cardiology/American Heart Association Task Force on Clinical Practice Guidelines and the Heart Failure Society of America. J Am Coll Cardiol 2017;70:776-803.

7 Weeda ER, Nguyen E, White CM. Role of ivabradine in the treatment of patients with cardiovascular disease. Ann Pharmacother 2016;50:475-85.

8 Tse S, Mazzola N, Ivabradine MN. Ivabradine (Corlanor) for heart failure: the first selective and specific I F inhibitor. P T 2015;40:810-4.

9 Benstoem C, Kalvelage C, Breuer T, et al. Ivabradine as adjuvant treatment for chronic heart failure. Cochrane Database Syst Rev 2020;11:CD013004.

10 Pei H, Miao W, Xie W-Z, et al. Ivabradine improves cardiac function and increases exercise capacity in patients with chronic heart failure. Int Heart J 2019;60:899-909.

11 Wetterslev J, Jakobsen JC, Gluud C. Trial sequential analysis in systematic reviews with meta-analysis. BMC Med Res Methodol 2017;17:39.

12 Keus F, Wetterslev J, Gluud C, et al. Evidence at a glance: error matrix approach for overviewing available evidence. BMC Med Res Methodol 2010;10:90.

13 Jakobsen JC, Wetterslev J, Winkel P, et al. Thresholds for statistical and clinical significance in systematic reviews with meta-analytic methods. BMC Med Res Methodol 2014;14:120.

14 Higgins JPT, Thompson SG. Quantifying heterogeneity in a meta-analysis. Stat Med 2002;21:1539-58.

15 Page MJ, McKenzie JE, Bossuyt PM, et al. The PRISMA 2020 statement: an updated guideline for reporting systematic reviews. BMJ 2021;372:n71.

16 Maagaard M, Nielsen EE, Gluud C. Ivabradine for coronary artery disease and/or heart failure: a protocol for a systematic review of randomised clinical trials with meta-analysis and trial sequential analysis. prospero 2018 CRD42018112082, 2018. Available: http://www.crd.york.ac.uk/ PROSPERO/display_record.php?ID=CRD42018112082

17 Higgins JP, Green S. Cochrane Handbook for systematic reviews of interventions, 2011. Available: www.cochrane-handbook.org

18 Lundh A, Lexchin J, Mintzes B, et al. Industry sponsorship and research outcome. Cochrane Database Syst Rev 2017;2:MR000033.

19 Review manager (RevMan), 2014. Available: https://training.cochrane. org/online-learning/core-software-cochrane-reviews/revman/revman-5download

20 Fox K, Ford I, Steg PG, et al. Ivabradine for patients with stable coronary artery disease and left-ventricular systolic dysfunction (beautiful): a randomised, double-blind, placebo-controlled trial. Lancet 2008;372:807-16.

21 Swedberg K, Komajda M, Böhm M, et al. Ivabradine and outcomes in chronic heart failure (shift): a randomised placebo-controlled study. Lancet 2010;376:875-85.

22 Thorlund K, Devereaux PJ, Wetterslev J, et al. Can trial sequential monitoring boundaries reduce spurious inferences from meta-analyses? Int J Epidemiol 2009;38:276-86.

23 Imberger G, Thorlund K, Gluud C, et al. False-Positive findings in Cochrane meta-analyses with and without application of trial sequential analysis: an empirical review. BMJ Open 2016;6:e011890.

24 Guyatt GH, Oxman AD, Vist GE, et al. Grade: an emerging consensus on rating quality of evidence and strength of recommendations. BMJ 2008;336:924-6.

25 Schünemann H, Brożek J, Guyatt G. Grade Handbook for grading quality of evidence and strength of recommendations, 2013. Available: https:// gdt.gradepro.org/app/handbook/handbook.html

26 Abdel HadyYA. Effects of adding ivabradine to optimal medical therapy in symptomatic patients with left ventricular systolic dysfunction secondary to ischemic or idiopathic dilated cardiomyopathy. Eur J Heart Fail

27 Abdel-Salam Z, Rayan M, Saleh A, et al. I(f) current inhibitor ivabradine in patients with idiopathic dilated cardiomyopathy: Impact on the exercise tolerance and quality of life. Cardiol J 2015;22:227-32.

28 Adamyan KG, Chilingarian AL, Astvacatryan AV. Addition of ivabradin further improves clinical status in patients with end stage heart failure and intolerance to ss-blockers. Circulation 2008;118:E341-2.

29 Schultz CJ, Moelker A, Piazza N, et al. Three dimensional evaluation of the aortic annulus using multislice computer tomography: are manufacturer's guidelines for sizing for percutaneous aortic valve replacement helpful? Eur Heart J 2010;31:849-56.

30 Adamyan KG, Tumasyan LR, Chilingaryan AL. Efficacy of longterm ivabradine therapy on prognosis, left and right heart functional parameters in patients with chronic heart failure and preserved left ventricular systolic function. European Heart Journal 2015;36:666-7.

31 Al Saadi T, Sallam M, Al Hashmi K, et al. PP166-Effect of carvedilol versus Carvedilol/Ivabradine combination on heart rate, quality of life, morbidity and mortality in patients with stable ischemic heart failure. Clin Ther 2013;35:e70.

32 Aroutunov GP, Aroutunov AG, Volkova AL. Prognosis of heart rate control on decompensated heart failure patients. Circulation 2008;118:E164.

33 Babushkina GV, Shaikhlislamova GI. [Ivabradine for Treatment of Heart Failure with Preserved Ejection Fraction]. Kardiologiia 2020;60:33-7.

34 Bansal S, Chaudhary M. Importance of serum sodium in ischemic heart failure with systolic dysfunction. European Journal of Heart Failure 2019;21:377.

35 Barillà $\mathrm{F}$, Pannarale G, Torromeo $\mathrm{C}$, et al. Ivabradine in patients with ST-elevation myocardial infarction complicated by cardiogenic shock: a preliminary randomized prospective study. Clin Drug Investig 2016;36:849-56. -

36 Bi J, Hou Y. Effect of ivabradine on serum NT-proBNP MMP-9 in patients with chronic heart failure. The Medical Forum 2020;24:1529-30.

37 Cao J, Li J. Effects of ivabradine on serum cyclophilin A and copeptin in patients with chronic congestive heart failure. Practical Geriatrics 2019;33:1217-20.

38 Cavusoglu Y, Mert KU, Nadir A. Dobutamine-induced increase in heart rate is blunted by ivabradine treatment in patients with acutely decompensated heart failure. European Heart Journal 2012;33:8.

39 Chaudhari MP, Chakraborty P, Pathak SP, et al. Effects of ivabradine on left ventricular function in patients with ischemic heart failure. Indian Heart J 2014;66:S83-4.

40 Chen G. Effect of ivabradine on serum NT-proBNP left ventricular ejection fraction and heart rate variability in patients with chronic heart failure. The Medical Forum 2020;24:2860-1.

41 Chen HX, Deng RS. Pharmacologic observation of ivabradine in the treatment of moderate to severe chronic heart failure combined with sinus tachycardia. Chinese Community Doctors 2021;37:16-17.

42 Chen XJ, JM L. Effectiveness of ivabradine combined with levosimendan for treating chronic heart failure. Frontiers of Medicine 2021;7:100.

43 Cheng LD, Jiang YC, Yan KL. Observation on curative efficacy and safety of ivabradine combined with routine treatment of chronic heart failure. Chinese Journal of Pharmacoepidemiology 2017;26:589-92.

44 Chumburidze SH, Khosroshvili L, Shelia G. Efficacy and safety of ivabradin treatment in patients with dilated cardiomyopathy. European Journal of Heart Failure 2013;12:S152.

45 Cong ZC. Evaluation of the efficacy of ivabradine in the treatment of chronic systolic heart failure. Chinese Practical Medicine 2018;13:113-4.

46 Deng YC. Efficacy and adverse reactions of ivabradine in the treatment of chronic heart failure. The World Clinical Medicine 2017;11:77.

47 Di T, Gao XL, Gao JY. Clinical study of ivabradine combined with metoprolol in the treatment of heart failure. Chinese Journal of Integrative Medicine on Cardio-Cerebrovascular Disease 2020;18:3613-6.

$48 \mathrm{Fu}$ Y, Wang WH, Lin HY. Effect of ivabradine on lipoprotein phospholipase A2 and cardiac function in heart failure patients with mid-range ejection fraction. Lingnan Journal of Emergency Medicine 2021;26:132-4. 
49 Gou HY, Chen YQ, XL W. Influence of ivabradine on 24-h heart rate, Btype natriuretic peptide and 6-minute walk distance in patients with acute decompensated systolic heart failure. Chin J Evid Based Cardiovasc Med 2017;9:348-50.

50 Guo Y, Zhang GS, Yan N. The effect of heart rate control on cardiac function of patients with heart failure. China Health Standard Management 2017;8:72-4.

51 HT H, GH X, Fang YF. The improvement of ventricular remodeling in patients with coronary heart disease and heart failure by ivabradine. Contemporary Medicine 2019;25:70-3.

$52 \mathrm{CJ} \mathrm{H}, \mathrm{CY}$ M. Analysis on clinical curative effect of ivabradine in the treatment of chronic systolic heart failure. Jiangsu Medicine 2017;12:34-6.

53 SD H, Cao MQ, XB L. Efficacy of ivabradine in treating acute myocardial infarction patients with heart failure. Jiangsu Medicine 2018;44:1428-31.

54 Huang J, Qian HY, ZC H. The effect of ivabradine on the leve LS of cystatin C in elderly patients with chronic heart failure. Chinese Journal Difficult and Complex cases 2017;16:545-9.

55 Komajda M, Isnard R, Cohen-Solal A, et al. Effect of ivabradine in patients with heart failure with preserved ejection fraction: the EDIFY randomized placebo-controlled trial. Eur J Heart Fail 2017; 19:1495-503.

56 Kosmala W, Holland D, Rojek A, et al. Beneficial effect of If-channel inhibition on exercise tolerance in heart failure with preserved ejection fraction. Eur Heart J 2013;34:183.

$57 \mathrm{Li} \mathrm{B}$. Effect of ivabradine combined with metoprolol in the treatment of chronic heart failure. Chin J Mod Drug Appl 2020;14:129-30.

58 JP L. Effect of sodium creatine phosphate combined with ivabradine in the treatment of chronic heart failure. Modern Practical Medicine 2018;30:356-7.

59 Li Q. Effects of ivabradine combined with carvedilol on cardiac function, serum NT-pro BNP and AngII levels in patients with chronic heart failure. J Sichuan Univ 2020;37:77-9.

60 Liu HZ, Li C, Zong WX. Effect of ivabradine on plasma H2S and BNP in patients with chronic pulmonary heart disease and heart failure. JNSMC 2019;34:270-2.

61 Liu YF. Clinical effect of ivabradine in the treatment of chronic systolic heart failure. Chinese Community Doctors 2020;36:34-6.

62 Liu YY, Zhang HB. Clinical effect of ivabradine in the treatment of heart failure with preserved ejection fraction : a randomized, double blind , controlled clinical study. Practical Journal of Cardiac Cerebral Pneumal and Vascular Disease 2020;28:16-20.

63 Lofrano-Alves MS, Issa VS, Biselli B, et al. Control of sinus tachycardia as an additional therapy in patients with decompensated heart failure (CONSTATHE-DHF): a randomized, double-blind, placebo-controlled trial. J Heart Lung Transplant 2016;35:1260-4.

$64 \mathrm{Lu}$ P. The effect of ivabradine on cardiac function and cardiac echocardiographic parameterss in patients with dilated cardiomyopathy. Yanbian University, 2019.

65 YH L, Shen WH. Influence of ivabradine combined with levosimendan on ventricular remodeling in senile patients with chronic heart failure. Chin Med Her 2020;17:123-6.

66 Luo S, Lin Z, Li J. Efficacy of Iavbredeen in treatment of very elderly patients with heart failure with mid-range ejection fraction. Journal of SNAKE 2021;33:44-6.

67 TL M, Han RD, Cao FL. Effects of ivabradine on myocardial microcirculation and levels of platelet parameters in patients with chronic pulmonary heart disease complicated with heart failure. Journal of Clinical and Experimental Medicine 2020;19:2156-60.

$68 \mathrm{Ma}$ Z. Ivabradine bisulfate for patients with moderate to severe chronic heart failure andleft ventricular systolic dysfunction [Master Degree of Medicine. University of Jinan, 2016.

69 Mansour S, Youssef A, Rayan M, et al. Efficacy of ivabradine in idiopathic dilated cardiomyopathy patients with chronic heart failure. Egypt Heart J 2011;63:79-85.

70 Manz M, Reuter M, Lauck G, et al. A single intravenous dose of ivabradine, a novel I(f) inhibitor, lowers heart rate but does not depress left ventricular function in patients with left ventricular dysfunction. Cardiology 2003;100:149-55.
71 Mao Q, Liang XL, Zhao XJ. Effect of ivabradine on chronic heart failure and its effect on biomarkers. Mod Med J 2018;46:1017-21.

72 Masi De Luca G, Palmisano P, Zaccaria M. Ivabradine improves physical performance and neurohormonal parameters in patients with diastolic heart failure. Circulation 2013:12816580.

73 Moiseev V, Potapenko A, Abdulasisov 0. Efficacy of ivabradine in patients with chronic heart failure after myocardial infarction. Eur J Heart Fail 2011;10:S234.

74 Nguyen L, Squara P, Amour J, et al. Intravenous ivabradine in low cardiac output syndrome after cardiac surgery treated by dobutamine: a phase II trial. Arch Cardiovasc Dis Suppl 2018;10:41.

75 Ordu S, Yildiz BS, Alihanoglu YI, et al. Effects of ivabradine therapy on heart failure biomarkers. Cardiol J 2015;22:501-9.

76 Pal N, Sivaswamy N, Mahmod M, et al. Effect of selective heart rate slowing in heart failure with preserved ejection fraction. Circulation 2015;132:1719-25.

77 Pan HY, Qian J, Pan M. Short-Term efficacy of ivabradine in patients with acute decompensated heart failure with reduced ejection fraction. Clinical Focus 2020;35:317-21.

78 Potapenko AV, Abdulazizov OS, Diachuk LI, et al. [Efficacy of ivabradin in combined treatment of patients with postinfarction systolic chronic cardiac failure]. Ter Arkh 2011;83:19-26.

79 Qi F, Liu B, Jin C. Study on effects of dibutyryl cyclic adenosine phosphate combined with ivabradine in the treatment of chronic heart failure. Medical Journal of National Defending Forces in Southwest China 2019;29:740-3.

80 Raja DC, Kapoor A, Sinha A, et al. Heart rate manipulation in dilated cardiomyopathy: assessing the role of ivabradine. Indian Heart $J$ 2018;70:246-51.

81 Sallam M, Al-Saadi T, Alshekaili L, et al. Impact of ivabradine on healthrelated quality of life of patients with ischaemic chronic heart failure. Curr Vasc Pharmacol 2016;14:481-6.

82 Sarullo FM, Fazio G, Puccio D, et al. Impact of "off-label" use of ivabradine on exercise capacity, gas exchange, functional class, quality of life, and neurohormonal modulation in patients with ischemic chronic heart failure. J Cardiovasc Pharmacol Ther 2010;15:349-55.

83 Shen LX, Wen JJ. Effect of ivabradine in the treatment of senile heart failure and its influence on NTproBNP , sST2 and galectin-3. Practical Pharmacy And Clinical Remedies 2018;21:526-9.

84 Sisakian H, Sargsyan T, Khachatryan A. Effect of selective heart rate reduction through sinus node If current inhibition on severely impaired left ventricular diastolic dysfunction in patients with chronic heart failure. Acta Cardiol 2016;71:317-22.

85 Song QY, Li M, Wang S. Effect of ivabradine on cardiac function and plasma N-terminal brain natriuretic peptide in elderly patients with heart failure and asthenia syndrome. J West China 2021;33:93-6.

86 DL S, Liao JX, Lin QF. Ivabradine hydrochloride tablets combined with metoprolol sustained-release tablets in the treatment of chronic heart failure. Jilin Med J 2020;41:1126-8.

87 DL S, Wei FQ, Deng MJ. Clinial efficacy of ivabradine combined with Zhigancao decoction in the treatment of chronic heart failure. Inner Mongolia Journal of Traditional Chinese Medicine 2020;39:5-7.

88 Sun JC. Effect of clinical application of ivabradine combined with metoprolol in the treatment of chronic heart failure. China Prac Med 2021;16:130-2.

89 Sun JK, Xue XC, LD H. Effect of ivabradine on chronic heart failure, Btype brain natriuretic peptide and N-terminal pro-B-type brain natriuretic peptide. China Medicine 2020;15:182-6.

90 Tang K, Zhao JH. Analysis of efficacy and safety on ivabradine combined with $\beta$-receptor blockers in the early treatment of chronic heart failure. Journal of Logistics University of PAP:Medical Sciences 2018;27:407-10.

91 Tarlovskaya E, Maksimchuk N, Kazakovtseva M. Clinical experience of treatment with ivabradine in patients with severe chronic heart failure. Eur J Heart Fail 2011;10:S32.

92 Tatarchenko IP, Pozdniakova NV, Biriuchenko MV, et al. [Clinical efficacy of ivabradin and nebivolol addition in combined treatment of ischemic heart disease patients with left ventricular dysfunction]. Ter Arkh 2008;80:40-4. 
93 Tsutsui H, Momomura S, Yamashina A, et al. Heart Rate Control With If Inhibitor, Ivabradine, in Japanese Patients With Chronic Heart Failure A Randomized, Double-Blind, Placebo-Controlled Phase II Study. Circ J 2016;80:668-76.

94 Tsutsui H, Momomura S-I, Yamashina A, et al. Efficacy and Safety of Ivabradine in Japanese Patients With Chronic Heart Failure - J-SHIFT Study. Circ J 2019;83:2049-60.

95 Tumasyan LR, Adamyan KG. Comparative efficacy of carvedilol and ivabradine in severe chronic heart failure of ischemic origin with baseline heart rate below and above 70 beat per minute. Eur Heart J 2009;30:427.

96 Tumasyan LR, Adamyan KG. Efficacy of long-term ivabradine therapy in severe systolic chronic heart failure patients with and without type 2 diabetes mellitus. Eur Heart J Cardiovasc Imaging 2016;17:12.

97 Tumasyan LR, Adamyan KG. Comparative efficacy of long-term digoxin and ivabradine therapy on prognosis, left and right heart functional parameters in patients with chronic heart failure and preserved ejection fraction. Eur J Heart Fail 2017;19:413.

98 Tumasyan LR, Adamyan KG. Comparative efficacy of long-term digoxin and ivabradine therapy on prognosis, left and right heart functional parameters in patients with chronic heart failure and mid-ranged ejection fraction. Eur J Heart Fail 2018;20:256.

99 Tumasyan LR, Adamyan KG, Chilingaryan AL. Efficacy of ivabradine therapy on right heart parameters and prognosis in patients with severe systolic chronic heart failure. Eur Heart J 2012;33:808.

100 Vatinian S, Gurgenyan S, Nikoghosyan K. Efficacy of addition of ivabradine to bisoprolol in patients with essential hypertension, coronary artery disease and reduced left ventricular systolic function. Eur Heart J 2015;36:672-3.

101 Wang AQ, Yang GJ, Yan SR. The efficacy of ivabradine in the treatment of patients with chronic heart failure. Journal of Heze Medical College 2019;31:30-2.

102 Wang FC. The effectiveness of ivabradine in elderly patients with chronic heart failure. Journal Of Clinical Medicine 2017;4:17790-1.

103 Wang GK. Study on the application value of ivabradine in vulnerable stage of heart failure. Journal of SNAKE 2020;32:437-9.

104 Wang JJ. Clinical observation of ivabradine combined with metoprolol in the treatment of chronic heart failure. Journal of Clinical Medicine 2017;4:13823.

105 Wang LJ, Liu FF, Sun XW. Effects of levosimendan combined with ivabradine hydrochloride on heart function, myocardial fibrosis markers and exercise tolerance in patients with chronic heart failure. Internal Medicine 2020;15:690-3.

106 Wang Q, Lin W. Clinical effect of ivabradine in treatment of patients with chronic heart failure. Acta Academiae Medicine QingDao University 2017;53:664-7.

107 Wang R. Effect of ivabradine on ventricular rate control in heart failure vulnerable period. Clinical Medical Research And Practice 2017;2:5-7.

108 Wang YH, Zhang Z, Wang J. Effects of ivabradine on cardiac function and NT-proBNP in patients with chronic heart failure. Acta Acad Med Weifang 2018;40:214-6.

109 Wei Y, Fu Q, ZJ L. Clinical effect and adverse reactions of ivabradine in the treatment of chronic heart failure. Clinical Research and Practice 2019;4:33-4.

110 Xia ZG, Yan J, Zhu XY. Effects of ivabradine tablets on heart function and serum catecholamine level in patients with heart failure. China pharmaceuticals 2016;25:20-3.

111 Xing ZM. Clinical efficacy of ivabradine Hydrochlorid combined with metoprolol in patients with chronic heart failure. China Continuing Medical Education 2018;10:112-4.

$112 \mathrm{Xu} \mathrm{Y}$, Shi R. Effect of ivabradine on IL-6 and TNF- $\alpha$ in patients with chronic heart failure. Journal of Logistics University of PAP 2019;28:40-2.

113 YQ X, Wang DF. Efficacy of ivabradine combined with Shensong Yangxin capsule on elderly patients with chronic heart failure and its effect on lipid metabolism, brain natriuretic peptide and cardiac function. Chinese Journal of Gerontology 2020;40:4932-4.

114 Xue D. The effect of ivabradine combined with metoprolol on heart rate variability and QT dispersion in patients with heart failure. China Medicine and Pharmacy 2020;10:85-7.
115 Yang WT, Lin XJ, Zheng Y. Clinical effect of ivabradine and its influence on hemodynamics and vascular endothelial function in patients with chronic heart failure. Chinese Journal of Health Laboratory Technology 2019;29:350.

116 Yang Z. Effectiveness of ivabradine in the treatment of acute myocardial infarction complicated with heart failure. Chinese Journal of Urban and Rural Industrial Hygiene 2019;31:24-6.

117 Clinical efficacy of ivabradine in the treatment of chronic systolic heart failure. "Emergency Medicine Clinical Academic Research Conference" meeting; 2016; Beijing.

118 RX Y, Li P, Li R. Effect of ivabradine on cardiac function and heart rate variability of ischemic cardiomyopathy patients. Chinese Journal ofCardiovascular Research 2017;15:1030-3.

$119 \mathrm{Yu}$ J. Analysis of the application of ivabradine in the treatment of chronic heart failure. Particularly Healthy 2018;15:197.

120 XB Y, Zhang HY, Xing DJ. Effects of ivabradine in the patients with chronic heart failure resulting from dilated cardiomyopathy. China Health Standard Management 2019;10:69-71.

121 Yue LB. Efficacy and safety of ivabradine sustained-release tablets in the treatment of elderly patients with chronic heart failure [master degree of medicine]. University of Jinan, 2016.

122 Zeng FC. Effectiveness of ivabradine in the treatment of chronic heart failure with sinus tachycardia. Chinese Journal of Misdiagnosis 2019;14:168-71.

123 Zeng XM, Dong XM, Feng H. Effects of ivabradine in the treatment of chronic heart failure and its influence on serum GDF-15 and sst2 levels in patients. Hainan Medical Journal 2019;30:2740-3.

124 Zhang J, Guo QZ LH. Effect of ivabradine in the treatment of acute exacerbation of chronic obstructive pulmonary disease with heart failure. Journal of Hainan Medical University 2019;25:1471-5.

125 Zhang J, Wang K. Effects of ivabradine on cardiac function and serum Galectin-3 , sST2 and NT-proBNP levels in patients with chronic heart failure. Chin J Clin Healthc 2021;24:59-62.

126 Zhang JH, Sun CF. Clinical effect of ivabradine on coronary heart disease combined with chronic obstructive pulmonary disease. Shaanxi Medical Journal 2019;48:256-8.

127 Zhang XJ. Effect of ivabradine combined with low-dose metoprolol tartrate in patients with chronic heart failure. Henan Medical Research 2019;28:1247-8.

128 Zhang Y, Zheng X. Analysis of clinical effect of ivabradine in adjuvant treatment of patients with chronic heart failure. Strait Pharmaceutical Journal 2020;32:134-5.

129 Zhang ZC. Effect of ivabradine combined with metoprolol on cardiac function and carotid intima-media thickness of patients with coronary heart disease complicated with heart failure. Medical Journal of Chinese People's Health 2020;32:11-13.

130 Zhao ST. The effect of ivabradine combined with levosimendan for treating chronic heart failure. China Health Care \& Nutrition 2020;30:285.

131 Zhou HT, JB B, Zhang DG. Effects of metoprolol combined with ivabradine on cardiac function in patients with severe heart failure complicated with sinus tachycardia. Cardio-Cerebrovasc Dis Prev Treat 2020;20:460-3.

132 Zhou Y. The effects of ivabradine on the heart rate variability and cardiac structure and function in chronic heart failure patients with mild-range ejection fraction. Southwest Medical University, 2019.

133 Fox K, Ferrari R, Tendera M, et al. Rationale and design of a randomized, double-blind, placebo-controlled trial of ivabradine in patients with stable coronary artery disease and left ventricular systolic dysfunction: the morBidity-mortality EvAlUaTion of the I(f) inhibitor ivabradine in patients with coronary disease and left ventricULar dysfunction (BEAUTIFUL) study. Am Heart J 2006;152:860-6.

134 Swedberg K, Komajda M, Böhm M, et al. Rationale and design of a randomized, double-blind, placebo-controlled outcome trial of ivabradine in chronic heart failure: the Systolic Heart Failure Treatment with the I(f) Inhibitor Ivabradine Trial (SHIFT). Eur J Heart Fail 2010;12:75-81. -

135 Servier. Effects of ivabradine on cardiovascular events in patients with moderate to severe chronic heart failure and left ventricular systolic dysfunction. A three-year international multicentre study 
(shift) ClinicalTrials2015. Available: https://clinicaltrials.gov/ct2/show/ NCT02441218 [Accessed 24 Feb 2020].

136 Servier. The beautiful study: effects of ivabradine in patients with stable coronary artery disease and left ventricular systolic dysfunction ClinicalTrials2005. Available: https://clinicaltrials.gov/ct2/show/ NCT00143507 [Accessed 24 Feb 2020].

137 Ekman I, Chassany 0, Komajda M, et al. Heart rate reduction with ivabradine and health related quality of life in patients with chronic heart failure: results from the shift study. Eur Heart $J$ 2011;32:2395-404.

138 De Luca GM, Palmisano P, Zaccaria M. Ivabradine improves physical performance and neurohormonal parameters in patients with diastolic heart failure. Circulation2013;128:16580.

139 Liana Tumasyan LR, Adamyan KG. Comparative efficacy of longterm digoxin and ivabradine therapy on prognosis, left and right heart functional parameters in patients with chronic heart failure and preserved ejection fraction. Eur J Heart Fail 2017;19:413.

140 Liana Tumasyan LR, Adamyan KG. Comparative efficacy of longterm digoxin and ivabradine therapy on prognosis, left and right heart functional parameters in patients with chronic heart failure and midranged ejection fraction. Eur J Heart Fail 2018;20:256.
141 Wood L, Egger M, Gluud LL, et al. Empirical evidence of bias in treatment effect estimates in controlled trials with different interventions and outcomes: meta-epidemiological study. BMJ 2008;336:601-5.

142 Schulz KF, Chalmers I, Hayes RJ, et al. Empirical evidence of bias. dimensions of methodological quality associated with estimates of treatment effects in controlled trials. JAMA 1995;273:408-12.

143 Savović J, Jones H, Altman D, et al. Influence of reported study design characteristics on intervention effect estimates from randomised controlled trials: combined analysis of meta-epidemiological studies. Health Technol Assess 2012;16:1-82.

144 Moher D, Pham B, Jones A, et al. Does quality of reports of randomised trials affect estimates of intervention efficacy reported in meta-analyses? Lancet 1998;352:609-13.

145 Kjaergard LL, Villumsen J, Gluud C. Reported methodologic quality and discrepancies between large and small randomized trials in meta-analyses. Ann Intern Med 2001;135:982-9.

146 Gluud LL. Bias in clinical intervention research. Am J Epidemiol 2006;163:493-501.

147 Maagaard M, Nielsen EE, Sethi NJ, et al. Effects of adding ivabradine to usual care in patients with angina pectoris: a systematic review of randomised clinical trials with meta-analysis and trial sequential analysis. Open Heart 2020;7:e01288. 